\title{
The Clinical Analysis on the postoperative complications of the Elderly Patients with the Oral and Maxillofacial Tumours
}

\author{
Guangping Sun ${ }^{1, a},{ }^{,}$Jingchun Huang ${ }^{1,{ }^{*}}$, Zhongyan Li $^{2}$ \\ 1 Department of Dentistry, The third Hospital of Jinan, Jinan, 250132; \\ ${ }^{2}$ The Department of Otorhinolaryngology, The third Hospital of Jinan, Jinan, 250132; \\ asun666@126.com
}

*Corresponding author huang68@126.com

Keywords: Surgery; Oral and maxillofacial tumours; Complications

\begin{abstract}
Objective: To observe the clinical curative effect of the surgical treatment of patients with the oral and maxillofacial tumours. Methods: From November 2012 to November 2012 the hospital treatment of 48 patients with the oral and maxillofacial tumours were randomized, the observation group of 24 patients with surgical treatment, the control group of 24 patients with radiation therapy, to compare two groups of patients in clinical symptoms before and after treatment. Results: The treatment group patients was $100 \%$ and 24 patients with the control treatment is $75.0 \%$ $(18 / 24)$; the treatment in patients with observation group is obviously higher than that of the control group $(\mathrm{P}<0.05)$. Two groups of adverse reactions are significant differences statistically significant $(\mathrm{P}<0.05)$. Conclusion: The surgical treatment of patients with oral and maxillofacial tumours reliable curative effect, high cure rate, is worth popularizing in clinical.
\end{abstract}

\section{Introduction}

The oral and maxillofacial tumours is one of the common malignant tumour of clinical, along with the increasing of the national health care consciousness and national health concept, in recent years, people begin to pay close attention to oral health problems, where the incidence of cancer and the detection rate increased year by year, become one of focuses on diseases of tumour diseases in oral and maxillofacial tumours, how to carcinoma, sarcoma is relatively small, from the lips, mouth, salivary glands, paranasal sinuses tumours, accounted for $6 \%$ of the whole body malignant tumour, causes the medical profession attaches great importance to. With the development of medical technology, method of treatment of oral and maxillofacial tumours is numerous, such as surgery, laser therapy, radiotherapy, etc. The clinical data showed that smoking and drinking alcohol is induced by oral and maxillofacial tumours, clinical treatment is difficult. Although radiotherapy can stable condition to a certain extent, but the treatment effect is not stable, easy to cause a series of complications, may increase again and again, is not conducive to treatment, a high incidence of adverse reactions. Clinical studies have found that according to the position of patients with lesions and pathological condition, choose the suitable treatment plan. For oral and maxillofacial tumours, early surgery treatment as soon as possible, by will tumour excision surgery, the analysis when necessary the cleaning technique, the prognosis of patients with improved significantly, but easy to damage in patients with oral tissue after tumour resection, induce dysfunction of oral and maxillofacial region. From November 2012 to November 2014 between hospitalization treatment of 48 patients with oral and maxillofacial tumours, now report as follows.

\section{Materials and Methods}

\section{Materials}

We select from November 2012 to November 2014, a total of 48 patients with oral and maxillofacial tumours, both by preoperative biopsy and postoperative histopathology examination confirmed, all for adenocarcinoma, before hospitalization of patients did not receive any treatment, and any hepatic and renal dysfunction or disease of heart head blood-vessel, etc. All patients were 
randomly divided into control group and observation group, each group of 24 cases, in which men 15 cases in control group, female 9 cases, patients age is 24-75, the average (49.6 \pm 3.6$)$ years of age, including labial cancer 8 cases, 6 cases of tongue cancer, tooth with carcinoma in 5 cases, parotid gland carcinoma 3 cases and submandibular gland carcinoma 2 cases, 11 cases with lymph node metastasis and lymph node metastases 13 cases; Male 14 cases in observation group, 10 cases of women, aged 25-73 years old, patients with average (48.7 \pm 4.2$)$, including a lip cancer 7 cases, 6 cases of tongue cancer, gingival carcinoma in 5 cases, 4 cases of parotid gland carcinoma and submandibular gland carcinoma 2 cases, including 12 cases with lymph node metastasis and 12 cases without lymph node metastasis. Two groups of patients in the general data, such as the gender, age and condition comparison, there were no statistically significant difference $(\mathrm{P}>0.05)$, comparable.

\section{Methods}

Observation group of 24 cases with surgical treatment, the cervical lymph nodes were lip cancer patients after anesthesia, gingival cancer and patients with tongue cancer undergoing routine joint radical surgery, closely observe the patient at the same time, in a critical condition to use chemotherapy to the patients cooperate with treatment. The treatment for 3 months is during this period to observe and record the change of the patients with cancer the status, physical condition, and adverse reactions. The control group is of 24 patients with radiation therapy, the use of radiation therapy, the dose of 60-70 Gy, emphasize radiotherapy positioning, treatment 1 year in a row. Compare two groups of patients in clinical symptoms before and after treatment.

\section{The outcome standard}

The powerfully: patients with oral cavity tumor disappear entirely, there is no risk of recurrence, the pain significantly reduce, at the same time of treatment satisfaction; Effective: patients with tumor shrinkage, pain significantly reduced, continued treatment can eliminate the bump, satisfied; Invalid: mass is not a big change, in patients with oral and facial pain, satisfied with the treatment of patients with low degree. Total effective rate $=$ (Obviously effective + Effective)/Total number of cases by $100 \%$.

\section{Statistical processing}

With mathematical statistics software SPSS19.0 for data collection and statistical analysis, and chi-square test, $\mathrm{P}<0.05$, significant difference has statistical significance.

\section{Results}

The treatment group patients was $100 \%$ and 24 patients with the control group treatment is $75.0 \%$ $(18 / 24)$; the treatment in patients with observation group is obviously higher than that of the control group $(\mathrm{P}<0.05)$. Two groups of adverse reactions are significant differences statistically significant $(\mathrm{P}<0.05)$. See Table 1 and Table 2.

Table 1: The effect comparison of two groups of patients

\begin{tabular}{cccccc}
\hline Group & $\mathrm{n}$ & $\begin{array}{c}\text { Obviously } \\
\text { effective }\end{array}$ & Effective & Invalid & $\begin{array}{c}\text { Total } \\
\text { effective } \\
\text { rate }\end{array}$ \\
\hline The treatment group & 24 & $16(66.67)$ & $8(33.33)$ & 0 & 100.0 \\
The control group & 24 & $8(33.33)$ & $10(41.67)$ & $6(25.0)$ & 75.0 \\
x 2 & & & & & 10.985 \\
P value & & & & & $<0.05$ \\
\hline
\end{tabular}


Table 2: The survival rate after treatment after 1-3 years in both groups

\begin{tabular}{ccccc}
\hline Group & $\mathrm{n}$ & One year & Two years & Three years \\
\hline The treatment group & 24 & $23(95.83)$ & $20(83.33)$ & $18(75.0)$ \\
The control group & 24 & $19(79.17)$ & $16(66.67)$ & $13(54.17)$ \\
x 2 & & 7.843 & 8.932 & 10.967 \\
P value & & $<0.05$ & $<0.05$ & $<0.05$ \\
\hline
\end{tabular}

\section{Discussion}

\section{The incidence}

The Oral and maxillofacial malignant tumor is a common malignant tumor, the sarcoma of the tumor is relatively small, common form for cancer. A significant portion of patients with cancer are squamous cell carcinoma, except there are a few of the gland carcinoma, lymph carcinoma, basal cell carcinoma and undifferentiated carcinoma. Position for the exposed parts, most of the oral cavity are cancer cells to form a long time there is a long process of precancerous lesions, therefore in clinical should pay attention to oral cancer early detection, early treatment. Because the precancerous lesions are likely to turn into a carcinoma, such as: pigment spots, chronic ulcers, such as skimming crack lesions. But it's also absolutely precancerous turn into cancer, which has many important inducing factors. In order to be able to prevent the occurrence of oral cancer, should take effective and positive way of symptomatic treatment of the lesions, the occurrence of chronic stimulation also banned all kinds of bad factors, such as smoking cessation, residual roots pull out, etc. In cases of suspected lesions than closely during the inspection follow-up, so that when an exception occurs in excision or biopsy.

\section{The anesthesia choice}

With the intraoperative vital signs and blood oxygen saturation changes, timely correction of anesthesia and surgery stimulation effect on the physiological function. After the surgery to treat patients with cough, swallowing reflex fully recover, fully conscious, and muscle tension well below can root out end tracheal intubations. Before the drawing tube net absorption trachea and nasal secretions, it is to reduce the occurrence of lung infection. Postoperative close monitoring should be in bed for tracheotomy apparatus and attractor rescue equipment and first aid such as medicine, in case of an accident when handled in time.

\section{The Operation characteristics}

There is a kind of injury, surgical treatment for patients before operation to the difficulty of the risk of surgery, surgical and patient tolerance of objective evaluation. In operation selection should be based on the physiological characteristics of elderly patients, pathology type, formulate reasonable operation scheme in accordance with the principle of tumor surgery. Radical was performed due to trauma, postoperative complications, more should be cautious when the choice, our group choose shoulder more feet hyoid retains the internal jugular vein, accessory nerve functional cleaning operation. Intraoperative as far as possible to shorten the operation time, reduce bleeding, strengthening postoperative care. The lesion resection and try to use draw simple suture, skin graft, and adjacent tissue flap repair surgery. The Pectorals major, and other large the flap for defect repair of oral and maxillofacial region has a large amount and high survival rate, but after the eradicative resection of oral and maxillofacial malignant tumor joint enforcement of large flap transplantation traumatic big, anesthesia and surgery time is too long, bigger blow on the elderly patients with the body, should be strictly grasp the surgical indications. 


\section{The postoperative complications}

In recent years, with the continuous improvement of people's living standard and health consciousness gradually strengthened, more and more people begin to pay attention to oral health, oral and maxillofacial tumors in clinical detection rate also increased year by year, of which the most common malignant tumor, and sarcoma is less, is now one of the common malignant tumor of clinically. Squamous cell carcinoma of the oral and maxillofacial tumors, most, followed by gland carcinoma, basal cell carcinoma, undifferentiated carcinoma and lymphatic carcinoma, develops in exposed parts, and process of precancerous lesions, so its early diagnosis and treatment of rehabilitation of patients and has the vital significance of life. Precancerous refers to the pathological changes could have evolved as malignant tumor, related literature reports, effective prevention of oral and maxillofacial tumors has important value, attaches great importance to the precancerous lesions (white spot, pigment spots, knit crack or chronic ulcer, etc.) of the prevention and treatment, and actively eliminate various pathogenesis of chronic stimulation factors (including smoking, drinking, etc.), the strict observation of condition change, necessary for biopsy or resection of lesion, in order to reduce the occurrence of cancer. Oral and maxillofacial malignant tumor is common with carcinoma, sarcoma is less. The vast majority of cancer is for squamous cell carcinoma, followed by gland carcinoma, and basal cell carcinoma and undifferentiated carcinoma, lymph carcinoma, etc. Most oral cancer in the exposed parts, and often precancerous process, this is good for oral cancer early detection, early treatment conditions. Precancerous lesions is a kind of can turn into cancer pathological changes, such as white spot, pigment spots, chronic ulcer, etc. Not all of these lesions will develop cancer, but also depends on other factors, finally evolved into cancer is only a few. In order to prevent the occurrence of oral cancer, should actively treatment of the lesions, and eliminate all kinds of bad chronic stimulation factors, such as smoking cessation, pull out the residual roots and residual crowns and remove bad repair, etc. The suspicious lesions should be closely followed up, if necessary, make biopsy or resection.

At present, the clinical treatment of oral and maxillofacial malignant tumor mainly adopts the surgery, radiotherapy or chemotherapy, including surgery treatment as the main means of clinical treatment, the line of cervical lymph nodes were performed at the same time, easy to cause tissue in patients with a wide range of serious defects, affect the appearance and normal function of oral and maxillofacial region, more blood osmotic solutions at the same time, combined with the surgical site, special operation method is complex, often leads to poor prognosis; And radiation and chemotherapy treatment is more serious adverse reactions, and after the treatment effect is not stable, more easy to cause the recurrence and death, therefore, to explore the diagnosis and treatment of reasonable method, by as much as possible to restore the normal function of the patients with oral and maxillofacial region, improve the patients quality of life, become the difficulty of the clinical treatment. In addition, in order to guarantee the curative effect of patients with surgery and postoperative quality of life, according to different patients should be appropriately and reconstructive surgery, increase the operation time and degree of trauma patients, have certain influence on the postoperative recovery of patients.

In the treatment of oral and maxillofacial malignant tumor, the tumor cut method usually causes oral tissue defects after the operation, serious impact on the patient's oral function and appearance. Therefore, in order to ensure the quality of surgery and postoperative quality of life, most of the patients chose to do surgery to repair the same period, so although increased the operation time and surgical trauma, and to a certain extent, affect the body's recovery, but surgical treatment in clinic is still very good results have been achieved. In this study, the clinical effect of treatment group than the control group. Because of oral and maxillofacial malignant tumor resection surgery of surgery, surgeons have higher technical quality and psychological quality, and the surgery for patients with trauma is bigger also, so require surgery also should have high professional quality of nurses. Nurses should not only know the process of operation, and familiar with the operation used in the various related equipment performance, try to shorten the bleeding time of the operation. Surgery before 1 day to complete the drug skin test, is preoperative preparation of skin preparation, blood, etc). Instruct patients pay attention to the body and ensure that patients sleep quality. At the same 
time such heavy belongs to the oral cavity jaw surgery and surgical operation, surgical trauma, high technical requirements; also have higher requirements for the nursing cooperation in. Nurses not only familiar with the head and neck anatomy and operation steps, but also be familiar with the operation of a variety of equipment performance, to create conditions for shorten the bleeding time. Tissue defect repair to old people at the same time, choose more conveniently, less injury, blood vessels, constant, the flap survival rate is high, the skin is empty inside and outside all can use (muscle) disc, such as the forearm back, leg, foot inside. Still have 1 day to do skin preparation, preoperative blood, drug skin test, etc., it cleans the body tell patients, guarantee the preoperative night sleep, appropriate use of sedatives when necessary, make the patient to get sufficient rest.

Because the elderly patients more systemic complications, blood vessels associated with atherosclerosis, operation risk is higher, the possibility of postoperative complications. Therefore, for older patients, line free tissue flap to repair should be cautious, to prevent postoperative vascular crisis. Drinking to assess preoperative patients, a large number of smoking history and presence of abnormal blood lipid, blood glucose and blood coagulation dysfunction. Especially for combination of elderly patients with severe hypertension, arteriosclerosis, unfavorable choose free tissue flap to repair. Flap surgery should be reasonable use of anticoagulant drugs, the use of portable ultrasound instrument examination, in order to find problems in time to save actively, improve the survival rate of tissue flap transplantation..

\section{Conclusion}

The correct assessment and actively control the complications, reasonable choice of anesthetic methods and operation program and strengthen the preoperative management, can make the most of the elderly oral facial tumor patient safety surgery treatment, thus the guarantee the patient recover smoothly, obtain ideal curative effect and prolong the life is very important. This group of data shows that the surgical treatment of patients with oral and maxillofacial tumours reliable curative effect, high cure rate, is worth popularizing in clinical.

\section{Acknowledgement}

This research was financially supported by the National Science Foundation.

\section{References}

[1] K. Wilson-d'Almeida, A. Karrow, M.-C. Bralet,N. Bazin, M.-C. [1] Mehta, Kinjal V,Lee, Haw Chou, Loh, James S Y. Mechanical thromboprophylaxis for patients undergoing hip fracture surgery[J]. Journal of Orthopaedic Surgery. 2012 (3)

[2] Niyati Parekh, Tomoko Okada, Grace L. Lu-Yao. Obesity, Insulin Resistance, and Cancer Prognosis: Implications for Practice for Providing Care among Cancer Survivors[J]. Journal of the American Dietetic Association. 2013(8)

[3] Giovanni Ghirlanda, Lorena Mancini. Current Perioperative Treatment of Patients with Type 1 and Type 2 Diabetes[J]. Clinics in Podiatric Medicine and Surgery. 2014 (3)

[4] Márta Ujpál, József Barabás, Ilona Kovalszky, György Szabó, Zsolt Németh, Katalin Gábris, Zsuzsanna Suba. A Preliminary Comparative Study of the Prognostic Implications of Type 2 Diabetes Mellitus for Patients With Primary Gingival Carcinoma Treated With Surgery and Radiation Therapy[J]. Journal of Oral and Maxillofacial Surgery. 2012 (3)

[5] W.BURGER,J. - M.CHEMNITIUS,G. D.KNEISSL,G.RÜCKER. Low - dose aspirin for secondary cardiovascular prevention - cardiovascular risks after its perioperative withdrawal versus bleeding risks with its continuation - review and meta - analysis[J]. Journal of Internal Medicine. 2013 (5)

[6] M J. Kovacs, C Kearon,M Rodger,D R. Anderson, A G.G. Turpie, S M. Bates,L Desjardins,J Douketis,S R. Kahn,S Solymoss,P S. Wells. Single-Arm Study of Bridging Therapy With 
Low-Molecular-Weight Heparin for Patients at Risk of Arterial Embolism Who Require Temporary Interruption of Warfarin[J]. Circulation. 2012 (12)

[7] Raymond S. Sinatra, Qiheng J. Shen, Thomas Halaszynski, Martha A. Luther,Yasser Shaheen. Preoperative Rofecoxib Oral Suspension as an Analgesic Adjunct After Lower Abdominal Surgery: The Effects on Effort-Dependent Pain and Pulmonary Function[J]. Anesthesia \& Analgesia. 2013 (1)

[8] John Butterworth and Curt D. Furberg. Improving Cardiac Outcomes After Noncardiac Surgery[J]. Anesthesia \& Analgesia. 2012 (3)

[9] Greet Van den Berghe, Pieter J. Wouters, Roger Bouillon, Frank Weekers, Charles Verwaest, Miet Schetz, Dirk Vlasselaers, Patrick Ferdinande, Peter Lauwers. Outcome benefit of intensive insulin therapy in the critically ill: Insulin dose versus glycemic control*[J]. Critical Care Medicine. $2013(2)$

[10] Kawalpreet Manku, Peter Bacchetti, Jacqueline M. Leung. Prognostic Significance of Postoperative In-Hospital Complications in Elderly Patients. I. Long-Term Survival[J]. Anesthesia \& Analgesia. 2012 (2) 